\title{
Synthesis, Antimicrobial Activity Studies and Molecular Property Predictions of Schiff Bases Derived from ortho-Vanillin
}

\author{
Barkın Berk ${ }^{\star 1}$, Merve Ertaş ${ }^{1}$, Sevde Nur Biltekin ${ }^{1}$ \\ 1 İstanbul Medipol University, School of Pharmacy, Kavacık Mah. Ekinciler Cad. No.19 Kavacık Kavşağı, \\ TR-34810, Beykoz, İstanbul, Turkey
}

\begin{abstract}
Schiff bases are known to possess anticancer, antibacterial, antifungal, antitubercular, anti-inflammatory, antibacterial and antimalarial properties. At the same time, in recent years, prediction of drug-likeness, molecular, absorption, distribution, metabolism, and excretion (ADME) properties using in silico techniques has become a standard procedure for the evaluation of clinical usable molecules. In this study, Schiff base structured 2-methoxy-6-\{[(2-alkyl/arylethyl)imino]methyl $\}$ phenol derivatives were synthesized from 3 -methoxysalicylaldehyde ( $o$-vanillin). The antibacterial and antifungal activities of these compounds were determined against Staphylococcus aureus, Escherichia coli, Candida albicans and Pseudomonas aeruginosa using agar-well diffusion and broth microdilution techniques. Further analysis was conducted using the in silico technique to predict the drug-likeness, molecular and ADME properties of these molecules. Among all the compounds, 2-\{[(4-fluorophenethyl)imino]methyl\}-6-methoxyphenol (Compound 9) exhibited the highest activity with good minimum inhibition concentration and radius of inhibition zone values against Candida albicans.
\end{abstract}

Keywords: Schiff bases, Antifungal, Candida albicans, Molecular properties, In silico

\section{INTRODUCTION}

Schiff bases (azomethines) are compounds containing structures of $\mathrm{R}-\mathrm{CH}=\mathrm{N}$ $\mathrm{R}^{\prime}\left(\mathrm{R}^{1} \mathrm{H}\right)$, where $\mathrm{R}$ and $\mathrm{R}^{\prime}$ can be either alkyl, aryl, cycloalkyl or heterocyclic groups ${ }^{1-3}$. These compounds are very well recognized and due to their unusual properties, they are used in a wide range of application areas including organic

${ }^{*}$ Corresponding author. Tel.; +90-216-6815364 Fax: +90-212-5317555.

E-mail address: bberk@medipol.edu.tr (Barkın Berk). 
and bioinorganic chemistry as common non-enzymatic/enzymatic intermediates, coordination and supramolecular chemistry as common ligands as well as biomedical applications and material sciences ${ }^{4-11}$. They have also been reported to show antibacterial ${ }^{12-17}$, antifungal ${ }^{14-16}$ and antitumoral ${ }^{18-19}$ activities.

Schiff bases can be synthesized by nucleophilic addition followed by dehydration reaction cascade between aliphatic or aromatic amines and active carbonyl compounds. Various derivatives of these compounds formed from the reaction between salicylaldehydes and amines act as cellular growth regulators for plants ${ }^{20}$ and have antibacterial ${ }^{21}$ and antimycotic ${ }^{22}$ activities. A wide range of different Schiff base ligands produced from ortho-hydroxyl substituted aromatic aldehydes have shown important coordinating properties for specific metal ions such as $\mathrm{Al}$ (III), Zn (II), Ag (II), Y (III), Co (II), Pb (II), Cu (II), Ni (II), Gd (III), Hg (II $^{23-28}$ and act as bidentate ligands for transitional metal ions ${ }^{29-33}$. At the same time, these compounds and/or their metal complexes have exhibited important anticancer and herbicidal properties ${ }^{34-35}$.

Similarly, a wide range of aldehyde and phenol structured secondary metabolites of plants isolated as crude extracts or oils have been studied for their potential antibacterial and antioxidant activities ${ }^{36-39}$. Ortho-vanillin ( $o$-vanillin) can be considered one of the several examples of these compounds with antibacterial and antifungal activity ${ }^{37,40-41}$ as well as irritant properties.

In the course of our study, we synthesized 2-methoxy-6-\{[(2-alkyl/arylethyl)imino]methyl\}phenol derivatives, Schiff bases of ortho-vanillin and 2-aryl/alkylethaneamines. The antibacterial and antifungal activities of the compounds were determined against Staphylococcus aureus, Escherichia coli, Candida albicans and Pseudomonas aeruginosa using agar-well diffusion and broth microdilution techniques. Furthermore, their absorption, distribution, metabolism, and excretion (ADME), drug-likeness and molecular properties were predicted using in silico techniques. Although Compounds 1, 3, 4, 7, 8, 13, 14 and 15 had been previously synthesized using different methods and investigated in other subjects ${ }^{42-49}$, in this study, we resynthesized them and performed a microbiological and prediction analysis to test their antibacterial and antifungal activities.

\section{METHODOLOGY}

\section{Chemistry}

All chemicals were purchased from Aldrich Chemical Co. (Steinheim, Germany). Melting points were determined with a Mettler-Toledo FP62 capillary melting point apparatus (Columbus, OH, USA) and uncorrected. IR spectra (KBr) were recorded on a PerkinElmer Spectrum One FT-IR spectrometer (Waltham, 
MA, USA) and ${ }^{1} \mathrm{H}-\mathrm{NMR}$ spectra were obtained by Bruker DPX-400, $400 \mathrm{MHz}$ High Performance Digital FT-NMR. All chemical shift values were recorded as $\delta(\mathrm{ppm})$. Mass spectra were recorded using an Agilent 1100 series LC/APCI/ MS 1946 G spectrometer in the negative ionization mode. The purity of the compounds was checked by thin-layer chromatography on silica gel-coated aluminum sheets (Merck, 1.005554, silica gel HF254-361, Type 60, 0.25 mm; Darmstadt, Germany). The elemental analyses were performed with a Leco CHNS 932 analyzer (Leco Corp., MI, USA) and were found to be within $\pm 0.4 \%$ of the theoretical values for $\mathrm{C}, \mathrm{H}$ and $\mathrm{N}$.

\section{General synthesis for Schiff derivatives}

Equimolar quantities (0.01 mol) of 3-methoxysalicylaldehyde and 2-aryl/alkylethaneamines were dissolved in methanol and stirred at room temperature for 10 to 120 min to obtain a clear solution using activated molecular sieves. Then, depending on the type of the substance, the solutions were either kept at room temperature or refrigerated overnight. The solutions that had precipitates were filtered, recrystallized, washed with cold methanol three times and dried in a vacuum desiccator. For the solutions that did not have any precipitates, column chromatography was performed using ethanol-ethyl acetate (1:1 v/v) as a mobile phase and the final compounds were obtained as either dark to light yellow solid crystals or yellow oils (Scheme 1).

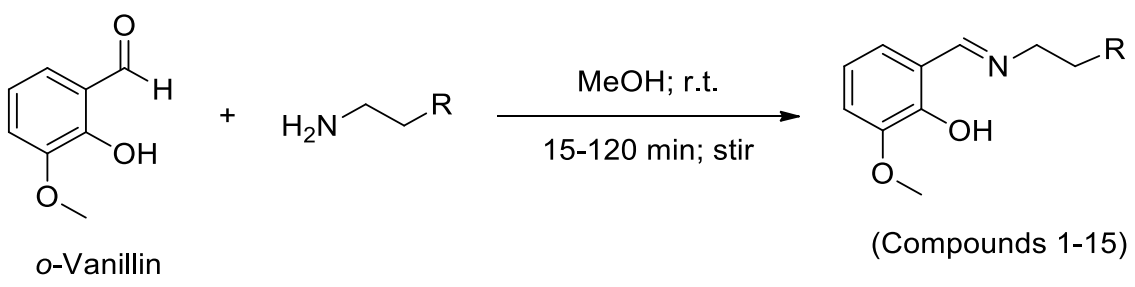

Scheme 1: Synthetic pathway followed for the preparation of 2-methoxy-6-\{[(2-alkyl/ arylethyl)imino]methyl\}phenol derivatives (Compounds 1-15)

\section{2-methoxy-6-(\{[2-(pyridin-2-yl)ethyl]imino\}methyl)phenol (Compound 1)}

Yield 80\%, yellow oil. IR (KBr) $\bar{v}_{\max }\left(\mathrm{cm}^{-1}\right): 3444(\mathrm{~s}, \mathrm{OH}), 3000(\mathrm{CH}$, aromatic), 2795 (CH, aliphatic), 1632 (C=N), 1254. ${ }^{1} \mathrm{H}-\mathrm{NMR}$ (400 MHz, DMSO-d6, 8): 3.2 ( $\left.\mathrm{t}, \mathrm{J}=7.10 \mathrm{~Hz}, 2 \mathrm{H}, \mathrm{Py}-\mathrm{CH}_{2}\right), 3.90\left(\mathrm{~s}, 3 \mathrm{H}, \mathrm{O}-\mathrm{CH}_{3}\right), 4.1$ (t, J=7.10 Hz, 2H, $\mathrm{CH}_{2}-$ $\mathrm{N}=\mathrm{C}), 5.30(\mathrm{~s}, 1 \mathrm{H}, \mathrm{OH}), 6.80-7.2(\mathrm{~m}, 3 \mathrm{H}, \mathrm{Ph}), 7.2-7.60,(\mathrm{~m}, 3 \mathrm{H}, \mathrm{Py}), 8.2(\mathrm{~s}, 1 \mathrm{H}$, $\mathrm{HC}=\mathrm{N}), 8.55(\mathrm{~d}, 1 \mathrm{H}, \mathrm{Py})$. MS 256.1 $\left(\mathrm{M}^{+}\right)$. Anal. calcd for $\mathrm{C}_{15} \mathrm{H}_{16} \mathrm{~N}_{2} \mathrm{O}_{2}: \mathrm{C}, 70.29 ; \mathrm{H}$, 6.29; N, 10.93. Found: C, 70.26; H, 6.31; N, 10.90 . 


\section{2-methoxy-6-(\{[2-(morpholin-4-yl)ethyl]imino\}methyl)phenol (Compound 2)}

Yield 70\%, yellow crystals, mp 38-39 ${ }^{\circ} \mathrm{C}$. IR $(\mathrm{KBr}) \bar{v}_{\text {max }}\left(\mathrm{cm}^{-1}\right): 3008(\mathrm{CH}$, aromatic), 2938 (CH, aliphatic), $1633(\mathrm{C}=\mathrm{N}), 1253 .{ }^{1} \mathrm{H}-\mathrm{NMR}$ (400 MHz, DMSO-d6,

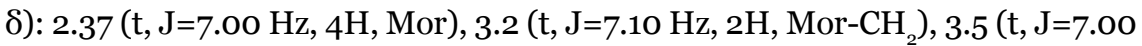
$\mathrm{Hz}, 4 \mathrm{H}, \mathrm{Mor}$ ), 3.90 (s, 3H, O-CH ${ }_{3}$ ), 4.0 (t, J=7.10 Hz, 2H, $\mathrm{CH}_{2}-\mathrm{N}=\mathrm{C}$ ), 5.35 (s, $1 \mathrm{H}, \mathrm{OH}), 6.90-7.1(\mathrm{~m}, 3 \mathrm{H}, \mathrm{Ph}), 8.25(\mathrm{~s}, 1 \mathrm{H}, \mathrm{HC}=\mathrm{N})$. MS 264.1 $\left(\mathrm{M}^{+}\right)$. Anal. calcd for $\mathrm{C}_{14} \mathrm{H}_{20} \mathrm{~N}_{2} \mathrm{O}_{3}$ : C, 63.62; H, 7.63; N, 10.60. Found: C, 63.60; H, 7.65; N, 10.59 .

\section{2-methoxy-6-(\{[2-(pyrrolidin-1-yl)ethyl]imino\}methyl)phenol (Compound 3)}

Yield 85\%, yellow oil. IR (KBr) $\bar{v}_{\max }\left(\mathrm{cm}^{-1}\right): 3429$ (s, OH), $2932(\mathrm{CH}$, aromatic), 2794 (CH, aliphatic), 1632 (C=N), 1254. ${ }^{1} \mathrm{H}-\mathrm{NMR}$ (400 MHz, DMSO-d6, 8): 1.7 (t, J=6.5 Hz, 4H, Pyr H), 2.60 (t, J=6.5 Hz, 4H, Pyr H), 2.80 (t, J=7.10 Hz, 2H, $\mathrm{CH}_{2}-\mathrm{Pyr}$ ), 3.76 (t, J=7.10 Hz, 2H, $\left.\mathrm{CH}_{2}-\mathrm{N}=\mathrm{C}\right), 3.90\left(\mathrm{~s}, 3 \mathrm{H}, \mathrm{O}-\mathrm{CH}_{3}\right), 5.3 \mathrm{O}(\mathrm{s}, 1 \mathrm{H}$, $\mathrm{OH}), 6.40-6.80(\mathrm{~m}, 3 \mathrm{H}, \mathrm{Ph}), 8.1(\mathrm{~s}, 1 \mathrm{H}, \mathrm{HC}=\mathrm{N})$. MS $248.2\left(\mathrm{M}^{+}\right)$. Anal. calcd for $\mathrm{C}_{14} \mathrm{H}_{20} \mathrm{~N}_{2} \mathrm{O}_{2}$ : C, 67.71; H, 8.12; N, 11.28. Found: C, 67.69; H, 8.15; N, 11.24.

\section{2-methoxy-6-(\{[2-(piperidin-1-yl)ethyl]imino\}methyl)phenol (Compound 4)}

Yield 75\%, yellow oil. IR (KBr) $\bar{v}_{\max }\left(\mathrm{cm}^{-1}\right): 3429(\mathrm{~s}, \mathrm{OH}), 2932(\mathrm{CH}$, aromatic),

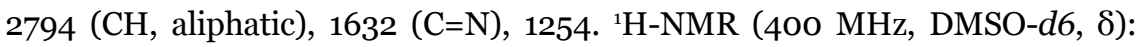
1.6- 1.8 (m, 6H, Pip H), 2.6o (t, J=7.00 Hz, 4H, Pip H), 2.80 (t, J=7.10 Hz, 2H, $\mathrm{CH}_{2}$-Pip), 3.76 (t, J=7.10 Hz, 2H, $\left.\mathrm{CH}_{2}-\mathrm{N}=\mathrm{C}\right), 3.90\left(\mathrm{~s}, 3 \mathrm{H}, \mathrm{O}-\mathrm{CH}_{3}\right), 5.35(\mathrm{~s}, 1 \mathrm{H}$, $\mathrm{OH}), 6.40-6.80(\mathrm{~m}, 3 \mathrm{H}, \mathrm{Ph}), 8.1(\mathrm{~s}, 1 \mathrm{H}, \mathrm{HC}=\mathrm{N})$. MS $262.2\left(\mathrm{M}^{+}\right)$. Anal. calcd for $\mathrm{C}_{15} \mathrm{H}_{22} \mathrm{~N}_{2} \mathrm{O}_{2}: \mathrm{C}, 68.67 ; \mathrm{H}, 8.45 ; \mathrm{N}, 10.68$. Found: C, 68.69; H, 8.48; N, 10.72.

\section{2-\{[(2-chlorophenethyl)imino]methyl\}-6-methoxyphenol (Compound 5)}

Yield 82\%, yellow crystals, mp 63-64 ${ }^{\circ} \mathrm{C}$. IR $(\mathrm{KBr}) \bar{v}_{\text {max }}\left(\mathrm{cm}^{-1}\right): 3010-3053(\mathrm{CH}$, aromatic), 2856-2931 (CH, aliphatic), $1632(\mathrm{C}=\mathrm{N}), 1253$, 735-790. ${ }^{1} \mathrm{H}-\mathrm{NMR}$ (400 MHz, DMSO-d6, $\delta$ ): 3.1 (t, J=7.10 Hz, 2H, $\mathrm{CH}_{2}-\mathrm{Ph}$ ), 3.80 (t, J=7.10 Hz, 2H, $\mathrm{CH}_{2}-$ $\mathrm{N}=\mathrm{C}), 3.90\left(\mathrm{~s}, 3 \mathrm{H}, \mathrm{O}-\mathrm{CH}_{3}\right), 5.35(\mathrm{~s}, 1 \mathrm{H}, \mathrm{OH}), 6.80-7.40(\mathrm{~m}, 7 \mathrm{H}, \mathrm{Ph}), 8.1(\mathrm{~s}, 1 \mathrm{H}$, $\mathrm{HC}=\mathrm{N})$. MS 289.1 $\left(\mathrm{M}^{+}\right)$. Anal. calcd for $\mathrm{C}_{16} \mathrm{H}_{16} \mathrm{ClNO}_{2}: \mathrm{C}, 66.32 ; \mathrm{H}, 5.57 ; \mathrm{N}, 4.83$. Found: C, 66.27; H, 5.60; N, 4.80 .

\section{2-\{[(2,4-dichlorophenethyl)imino]methyl\}-6-methoxyphenol (Compound 6)}

Yield 70\%, yellow crystals, mp 103-104 ${ }^{\circ} \mathrm{C}$. IR (KBr) $\bar{v}_{\max }\left(\mathrm{cm}^{-1}\right): 3005-3089(\mathrm{CH}$, aromatic), 2831-2961 (CH, aliphatic), $1633(\mathrm{C}=\mathrm{N}), 1253,702-710,819-867 .{ }^{1} \mathrm{H}-$ 
NMR (400 MHz, DMSO-d6, 8): 3.1 (t, J=7.10 Hz, 2H, - $\mathrm{CH}_{2}-\mathrm{Ph}$ ), 3.84 (t, J=7.10 $\left.\mathrm{Hz}, 2 \mathrm{H}, \mathrm{CH}_{2}-\mathrm{N}=\mathrm{C}\right), 3.90\left(\mathrm{~s}, 3 \mathrm{H}, \mathrm{O}-\mathrm{CH}_{3}\right), 5.30(\mathrm{~s}, 1 \mathrm{H}, \mathrm{OH}), 6.80-7.30(\mathrm{~m}, 6 \mathrm{H}$, $\mathrm{Ph}), 8.1(\mathrm{~s}, 1 \mathrm{H}, \mathrm{HC}=\mathrm{N})$. MS $323.1\left(\mathrm{M}^{+}\right)$. Anal. calcd for $\mathrm{C}_{16} \mathrm{H}_{15} \mathrm{Cl}_{2} \mathrm{NO}_{2}: \mathrm{C}, 59.28 ; \mathrm{H}$, 4.66; N, 4.32. Found: C, 59.24; H, 4.69; N, 4.30.

\section{2-\{[(3-chlorophenethyl)imino]methyl\}-6-methoxyphenol (Compound 7)}

Yield 90\%, yellow crystals, mp 39-40 ${ }^{\circ} \mathrm{C}$. IR (KBr) $\bar{v}_{\max }\left(\mathrm{cm}^{-1}\right): 3009-3060(\mathrm{CH}$, aromatic), 2853-2936 (CH, aliphatic), $1633(\mathrm{C}=\mathrm{N}), 1254,780-731,774-838 .{ }^{1} \mathrm{H}-$ NMR (4Oo MHz, DMSO-d6, 8): 2.98 (t, J=7.10 Hz, 2H, CH $-\mathrm{Ph}$ ), 3.80 (t, J=7.10 $\mathrm{Hz}, 2 \mathrm{H}, \mathrm{CH}_{2}-\mathrm{N}=\mathrm{C}$ ), 3.88 (s, $3 \mathrm{H}, \mathrm{O}-\mathrm{CH}_{3}$ ), 5.30 (s, $\left.1 \mathrm{H}, \mathrm{OH}\right), 6.70-7.20(\mathrm{~m}, 7 \mathrm{H}, \mathrm{Ph}$ ), $8.1(\mathrm{~s}, 1 \mathrm{H}, \mathrm{HC}=\mathrm{N})$. MS 289.1 $\left(\mathrm{M}^{+}\right)$. Anal. calcd for $\mathrm{C}_{16} \mathrm{H}_{16} \mathrm{ClNO}_{2}: \mathrm{C}, 66.32 ; \mathrm{H}, 5.57$; N, 4.83. Found: C, 66.28; H, 5.61; N, 4.81.

\section{2-\{[(2-fluorophenethyl)imino]methyl\}-6-methoxyphenol (Compound 8)}

Yield 83\%, yellow crystal, mp 71-72 ${ }^{\circ} \mathrm{C}$. IR $(\mathrm{KBr}) \bar{v}_{\text {max }}\left(\mathrm{cm}^{-1}\right): 3010-3053(\mathrm{CH}$, aromatic), 2856-2931 (CH, aliphatic), $1632(\mathrm{C}=\mathrm{N}), 1253$, 735-790. ${ }^{1} \mathrm{H}-\mathrm{NMR}$ (400 MHz, DMSO-d6, $\delta$ ): 3.04 (t, J=7.10 Hz, 2H, $\mathrm{CH}_{2}-\mathrm{Ph}$ ), 3.84 (t, J=7.10 Hz, 2H, $\left.\mathrm{CH}_{2}-\mathrm{N}=\mathrm{C}\right), 3.90\left(\mathrm{~s}, 3 \mathrm{H}, \mathrm{O}-\mathrm{CH}_{3}\right), 5.3 \mathrm{O}(\mathrm{s}, 1 \mathrm{H}, \mathrm{OH}), 6.80-7.20(\mathrm{~m}, 7 \mathrm{H}, \mathrm{Ph}), 8.1$ $(\mathrm{s}, 1 \mathrm{H}, \mathrm{HC}=\mathrm{N})$. MS 273.1 $\left(\mathrm{M}^{+}\right)$. Anal. calcd for $\mathrm{C}_{16} \mathrm{H}_{16} \mathrm{FNO}_{2}: \mathrm{C}, 70.31 ; \mathrm{H}, 5.90 ; \mathrm{N}$, 5.12. Found: C, 70.28; H, 5.93; N, 5.09.

\section{2-\{[(4-fluorophenethyl)imino]methyl\}-6-methoxyphenol (Compound 9)}

Yield 70\%, yellow oil. IR (KBr) $\bar{v}_{\text {max }}\left(\mathrm{cm}^{-1}\right): 3427(\mathrm{~s}, \mathrm{OH}), 2941-3058(\mathrm{CH}$, aromatic), 2771-2827 (Al. CH), $1632\left(\mathrm{C}=\mathrm{N}\right.$ ), 1254, 736-780. ${ }^{1} \mathrm{H}-\mathrm{NMR}$ (400 MHz,

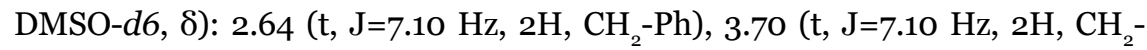
$\mathrm{N}=\mathrm{C}), 3.90\left(\mathrm{~s}, 3 \mathrm{H}, \mathrm{O}-\mathrm{CH}_{3}\right), 5.30(\mathrm{~s}, 1 \mathrm{H}, \mathrm{OH}), 6.80-7.10(\mathrm{~m}, 7 \mathrm{H}, \mathrm{Ph}), 8.1(\mathrm{~s}, 1 \mathrm{H}$, $\mathrm{HC}=\mathrm{N})$. MS $273.1\left(\mathrm{M}^{+}\right)$. Anal. calcd for $\mathrm{C}_{16} \mathrm{H}_{16} \mathrm{FNO}_{2}$ : C, 70.31; H, 5.90; N, 5.12. Found: C, 70.29; H, 5.94; N, 5.10.

\section{2-(\{[2-(dimethylamino)ethyl]imino\}methyl)-6-methoxyphenol (Compound 10)}

Yield 70\%, yellow oil. IR ( $\mathrm{KBr}) \bar{v}_{\max }\left(\mathrm{cm}^{-1}\right): 3433(\mathrm{~s}, \mathrm{OH}), 2941$ (CH, aromatic), 2772-2821 (CH, aliphatic), $1633(\mathrm{C}=\mathrm{N}), 1254,736-780 .{ }^{1} \mathrm{H}-\mathrm{NMR}$ (400 MHz, DMSO-d6, 8): $2.3\left(\mathrm{~s}, 6 \mathrm{H}, \mathrm{CH}_{3}-\mathrm{N}\right.$ ), 3.70 (t, J=7.10 Hz, $2 \mathrm{H}, \mathrm{CH}_{2}-\mathrm{N}$.), 3.80 (t, $\left.\mathrm{J}=7.10 \mathrm{~Hz}, 2 \mathrm{H}, \mathrm{CH}_{2}-\mathrm{N}=\mathrm{C}\right), 3.90\left(\mathrm{~s}, 3 \mathrm{H}, \mathrm{O}-\mathrm{CH}_{3}\right), 5.35(\mathrm{~s}, 1 \mathrm{H}, \mathrm{OH}), 6.80-7.10(\mathrm{~m}$, $3 \mathrm{H}, \mathrm{Ph}), 8.1(\mathrm{~s}, 1 \mathrm{H}, \mathrm{HC}=\mathrm{N})$. MS 222.0 $\left(\mathrm{M}^{+}\right)$. Anal. calcd for $\mathrm{C}_{12} \mathrm{H}_{18} \mathrm{~N}_{2} \mathrm{O}_{2}: \mathrm{C}, 64.84$; H, 8.16; N, 12.6o. Found: C, 64.81; H, 8.19; N, 12.58 . 


\section{2-(\{[2-(diethylamino)ethyl]imino\}methyl)-6-methoxyphenol (Compound 11)}

Yield 88\%, yellow oil. IR (KBr) $\bar{v}_{\max }\left(\mathrm{cm}^{-1}\right): 3435$ (s, OH), 2968 (CH, aliphatic), $1631\left(\mathrm{C}=\mathrm{N}\right.$ ), $1253 .{ }^{1} \mathrm{H}-\mathrm{NMR}$ (400 MHz, DMSO-d6, 8): 1.00 (t, J=8 Hz, 6H, $\mathrm{CH}_{3}-$ $\mathrm{CH}_{2}-\mathrm{N}-$ ), 2.40 (q, J=8 Hz, $4 \mathrm{H}, \mathrm{CH}_{3}-\mathrm{CH}_{2}-\mathrm{N}-$ ), 2.72 (t, J=7.10 Hz, $2 \mathrm{H},-\mathrm{CH}_{2}-\mathrm{N}$.), $3.65\left(\mathrm{t}, \mathrm{J}=7.10 \mathrm{~Hz}, 2 \mathrm{H}, \mathrm{CH}_{2}-\mathrm{N}=\mathrm{C}\right), 3.90\left(\mathrm{~s}, 3 \mathrm{H}, \mathrm{O}-\mathrm{CH}_{3}\right), 5.3 \mathrm{O}(\mathrm{s}, 1 \mathrm{H}, \mathrm{OH}), 6.4 \mathrm{O}-$ 7.10 (m, $3 \mathrm{H}, \mathrm{Ph}), 8.1(\mathrm{~s}, 1 \mathrm{H}, \mathrm{HC}=\mathrm{N})$. $\mathrm{MS} 250.2\left(\mathrm{M}^{+}\right)$. Anal. calcd for $\mathrm{C}_{14} \mathrm{H}_{22} \mathrm{~N}_{2} \mathrm{O}_{2}$ : C, 67.17; H, 8.86; N, 11.19. Found: C, 67.14; H, 8.89; N, 11.18 .

\section{2-\{[(2-chloroethyl)imino]methyl\}-6-methoxyphenol (Compound 12)}

Yield 88\%, yellow oil. IR (KBr) $\bar{v}_{\max }\left(\mathrm{cm}^{-1}\right): 3418$ (s, OH), $2965(\mathrm{CH}$, aliphatic), $1651\left(\mathrm{C}=\mathrm{N}\right.$ ), $1256 .{ }^{1} \mathrm{H}-\mathrm{NMR}$ (400 MHz, DMSO-d6, 8):3.60 (t, 2H, $-\mathrm{CH}_{2}-\mathrm{Cl}$ ), 3.83 (t, $2 \mathrm{H},-\mathrm{CH}_{2}-\mathrm{N}=\mathrm{C}$ ) $, 3.90\left(\mathrm{~s}, 3 \mathrm{H}, \mathrm{OCH}_{3}\right), 5.30(\mathrm{~s}, 1 \mathrm{H}, \mathrm{OH}), 6.5 \mathrm{O}-7.10(\mathrm{~m}, 3 \mathrm{H}, \mathrm{Ph})$, $8.1(\mathrm{~s}, 1 \mathrm{H}, \mathrm{HC}=\mathrm{N})$. MS 213.1 $\left(\mathrm{M}^{+}\right)$. Anal. calcd for $\mathrm{C}_{10} \mathrm{H}_{12} \mathrm{ClNO}_{2}: \mathrm{C}, 56.21 ; \mathrm{H}, 5.66$; N, 6.56. Found: C, 56.18; H, 5.70; N, 6.54.

\section{2-methoxy-6-(\{[2-(piperazin-1-yl)ethyl]imino\}methyl)phenol (Compound 13)}

Yield 75\%, yellow oil. IR (KBr) $\bar{v}_{\max }\left(\mathrm{cm}^{-1}\right): 3427$ (s, OH), $2938(\mathrm{CH}$, aromatic), 2830 (CH, aliphatic), 1633 (C=N), 1254. ${ }^{1} \mathrm{H}-\mathrm{NMR}$ (400 MHz, DMSO-d6, 8): 2.0 (s, 1H, Ppz NH), 2.48 (t, 4H, Ppz H), 2.65 (t, 4H, Ppz H), 2.72 (t, 2H, $\mathrm{CH}_{2}-\mathrm{Ppz}$ ), $3.83\left(\mathrm{t}, 2 \mathrm{H}, \mathrm{J}=7.10 \mathrm{~Hz}, \mathrm{CH}_{2}-\mathrm{N}=\mathrm{C}\right), 3.90\left(\mathrm{~s}, 3 \mathrm{H}, \mathrm{O}_{-} \mathrm{CH}_{3}\right), 5.35(\mathrm{~s}, 1 \mathrm{H}, \mathrm{OH}), 6.5 \mathrm{O}-$ $7.90(\mathrm{~m}, 3 \mathrm{H}, \mathrm{Ph}), 8.1(\mathrm{~s}, 1 \mathrm{H}, \mathrm{HC}=\mathrm{N})$. MS $263.2\left(\mathrm{M}^{+}\right)$. Anal. calcd for $\mathrm{C}_{14} \mathrm{H}_{21} \mathrm{~N}_{3} \mathrm{O}_{2}$ : C, 63.85; H, 8.04; N, 15.96. Found: C, 63.82; H, 8.07; N, 15.93.

\section{2-(\{[2-(1H-imidazol-2-yl)ethyl]imino\}methyl)-6-methoxyphenol (Compound 14)}

Yield 74\%, yellow oil. IR (KBr) $\bar{v}_{\max }\left(\mathrm{cm}^{-1}\right): 3437(\mathrm{~s}, \mathrm{OH}), 3005-3089(\mathrm{CH}$, aromatic), 2831-2961 (CH, aliphatic), $1635(\mathrm{C}=\mathrm{N}), 1254 .{ }^{1} \mathrm{H}-\mathrm{NMR}$ (400 MHz, DMSO-d6, 8): 2.90 (t, J=7.10 Hz, 2H, - $\mathrm{CH}_{2}$-Imd) 3.83 (t, 2H, J=7.10 Hz, $\mathrm{CH}_{2}-\mathrm{N}=\mathrm{C}$ ), $3.90\left(\mathrm{~s}, 3 \mathrm{H}, \mathrm{OCH}_{3}\right), 5.35(\mathrm{~s}, 1 \mathrm{H}, \mathrm{OH}), 6.60-7.30(\mathrm{~m}, 5 \mathrm{H}, \mathrm{Ph}$ and $\mathrm{Imd}), 8.1$ (s, $1 \mathrm{H}, \mathrm{HC}=\mathrm{N}), 13.00(1 \mathrm{H}, \mathrm{Imd}$. $\mathrm{NH})$. MS 245.1 $\left(\mathrm{M}^{+}\right)$. Anal. calcd for $\mathrm{C}_{13} \mathrm{H}_{15} \mathrm{~N}_{3} \mathrm{O}_{2}: \mathrm{C}$, 63.66; H, 6.16; N, 17.13. Found: C, 63.63; H, 6.19; N, 17.10.

\section{2-methoxy-6-[(phenethylimino)methyl]phenol (Compound 15)}

Yield 88\%, yellow crystal, mp 76-77 ${ }^{\circ} \mathrm{C}$. IR (KBr) $\bar{v}_{\max }\left(\mathrm{cm}^{-1}\right): 3023-3085(\mathrm{CH}$, aromatic), 2859-2996 (CH, aliphatic), $1627(\mathrm{C}=\mathrm{N}), 1253 \cdot{ }^{1} \mathrm{H}-\mathrm{NMR}$ (400 $\mathrm{MHz}$, DMSO-d6, $\delta$ ): 3.0 (t, J=7.10 Hz, 2H, $\mathrm{CH}_{2}-\mathrm{Ph}$ ), 3.84 (t, J=7.10 Hz, 2H, $\mathrm{CH}_{2}-\mathrm{N}=\mathrm{C}$ ), $3.90\left(\mathrm{~s}, 3 \mathrm{H}, \mathrm{OCH}_{3}\right), 5.30(\mathrm{~s}, 1 \mathrm{H}, \mathrm{OH}), 6.80-7.40(\mathrm{~m}, 8 \mathrm{H}, \mathrm{Ph}), 8.1(\mathrm{~s}, 1 \mathrm{H}, \mathrm{HC}=\mathrm{N})$. MS 255.1 $\left(\mathrm{M}^{+}\right)$. Anal. calcd for $\mathrm{C}_{16} \mathrm{H}_{17} \mathrm{NO}_{2}$ : C, 75.27; H, 6.71; N, 5.49. Found: C, 75.25; H, 6.74; N, 5.46 . 


\section{Microbiological Screening}

The following test microorganisms were obtained from LGC Standards GmbH (Wesel, Germany): Staphylococcus aureus (S. aureus) ATCC 25923, Escherichia coli (E. coli) ATCC 25922, Pseudomonas aeruginosa (P. aeruginosa) ATCC 27853, and Candida albicans (C. albicans) ATCC 60193. All the synthesized compounds were dissolved in dimethyl sulphoxide (DMSO) to prepare a stock solution at $10 \mathrm{mg} / \mathrm{mL}$.

\section{Agar-well diffusion method}

An adapted simple susceptibility screening based on agar-well diffusion was used $^{50-52}$. Each bacterium was suspended in a Mueller-Hinton broth (MHB) (Difco, Detroit, MI, USA) and the fungal sample, C. albicans, was suspended in a Sabouraud Agar Modified medium (BD 274720) for pre-incubation at $35^{ \pm 2}{ }^{\circ} \mathrm{C}$. Then, all the microorganisms were diluted until they approximately matched the turbidity of $0.5 \mathrm{McFarland}$ standard $\left(1-2 \times 10^{8} \mathrm{cfu} / \mathrm{mL}\right)$. Later, they were floodinoculated onto the surface of Mueller-Hinton (MH) and Sabouraud dextrose (SD) agars and dried. Five-millimeter diameter wells were cut from the agar using a sterile cork borer, and $20 \mu \mathrm{L}$ of the stock substances was delivered into the wells. The plates were incubated for $16-24 \mathrm{~h}$ at $35 \pm 2{ }^{\circ} \mathrm{C}$. Antibacterial activity was evaluated by measuring the radius of the inhibition zone against the test organism using a digital caliper. $o$-vanillin $(10 \mathrm{mg} / \mathrm{mL})$, ampicillin $(10 \mu \mathrm{g} / \mathrm{mL})$ and fluconazole $(5 \mu \mathrm{g} / \mathrm{mL})$ were used as standards. DMSO diluted to 1:10 was used as the solvent control to ensure that it did not have any effect on bacterial growth.

\section{Broth microdilution method}

The minimal inhibition concentration (MIC) values $(\mu \mathrm{g} / \mathrm{mL})$ for the organisms were determined using the methods recommended by the Clinical and Laboratory Standards Institute (CLSI) guidelines ${ }^{53-54}$. The antimicrobial effects of the substances against the $S$. aureus, $E$. coli and $C$. albicans microorganisms were tested quantitatively in broth media using double dilution. The broth microdilution method was not performed on $P$. aeruginosa since none of the compounds showed inhibition zones with the agar well diffusion method. The antibacterial and antifungal assays were performed in an $\mathrm{MH}$ broth (Difco) at $\mathrm{pH} 7.3$ and a buffered yeast nitrogen base (Difco) at pH 7.0, respectively. MIC was defined as the lowest concentration with no bacterial or fungal growth. Ampicillin $(10 \mu \mathrm{g} / \mathrm{mL})$ and fluconazole $(10 \mu \mathrm{g} /$ $\mathrm{mL}$ ) were prepared as stocks, then diluted in a range from 10 to $0.5 \mu \mathrm{g} / \mathrm{mL}$ using DMSO and tested as standard antibacterial and antifungal drugs, respectively. The tested dilutions ranged from 128 to $0.5 \mu \mathrm{g} / \mathrm{mL}$ using DMSO as the solvent for all compounds. The control samples prepared with the amounts of DMSO used in the dilutions did not show any inhibitory activity under these conditions. 


\section{Prediction of drug-likeness, molecular and ADME properties}

All the molecules were prepared in $3 \mathrm{D}$ using the LigPrep module of Maestro (Schrodinger Inc.). ADME properties (46 molecular descriptors) were calculated using the QikProp program (Schrödinger 2015-3) in the normal mode. QikProp generates physically relevant descriptors and uses them to perform ADME predictions. An overall ADME-compliance score, the drug-likeness parameter (indicated by \#stars), was used to assess the pharmacokinetic profiles of the compounds. The \#stars parameter (ranging from o to 5 ) indicates the number of property descriptors computed by QikProp that fall outside the optimum range of values for $95 \%$ of known drugs.

The predicted descriptors were: central nervous system (CNS) activity ( -2 for inactive to +2 for active); octanol/water partition coefficient, $\log \mathrm{Po} / \mathrm{w}(-2.0$ to 6.5); $\mathrm{IC}_{50}$ value for the block of HERG $\mathrm{K}^{+}$channels, $\log H E R G$ (concern $<-5$ ); Caco-2 cell membrane permeability in $\mathrm{nm} \mathrm{s}^{-1}$, PCaco $(:<5$ low to $>100$ high);

Table 1: Antimicrobial activity of compounds using the microdilution (MIC, $\mu \mathrm{g} / \mathrm{mL}$ ) and agar well diffusion methods (diameter of inhibition zones in $\mathrm{mm}$ ) ${ }^{\mathrm{a}}$

Minimal inhibition concentration $(\mu \mathrm{g} / \mathrm{mL})$ and diameter of inhibition zones $(\mathrm{mm}) \mathrm{a}$

\begin{tabular}{|l|l|l|l|l|}
\hline Compound & S. aureus & E. coli & C. albicans & P. aeroginosa \\
\hline $\mathbf{1}$ & $>128(0)$ & $>128(1)$ & $16(17)$ & $(0)$ \\
\hline $\mathbf{2}$ & $>128(0)$ & $>128(2)$ & $16(17)$ & $(0)$ \\
\hline $\mathbf{3}$ & $32(11)$ & $>128(0)$ & $16 .(17)$ & $(0)$ \\
\hline $\mathbf{4}$ & $16 .(15)$ & $32 .(7)$ & $64 .(22)$ & $(0)$ \\
\hline $\mathbf{5}$ & $>128(0)$ & $>128(0)$ & $64(14)$ & $(0)$ \\
\hline $\mathbf{6}$ & $>128(0)$ & $>128 .(0)$ & $>128 .(14)$ & $(0)$ \\
\hline $\mathbf{7}$ & $>128(0)$ & $>128(3)$ & $>128(14)$ & $(0)$ \\
\hline $\mathbf{8}$ & $>128(0)$ & $>128(3)$ & $16(21)$ & $(0)$ \\
\hline $\mathbf{9}$ & $32(13)$ & $16(9)$ & $8 .(25)$ & $(0)$ \\
\hline $\mathbf{1 0}$ & $32 .(11)$ & $64(4)$ & $16(21)$ & $(0)$ \\
\hline $\mathbf{1 1}$ & $32(11)$ & $>128(2)$ & $32(17)$ & $(0)$ \\
\hline $\mathbf{1 2}$ & $>128(0)$ & $64(5)$ & $16(22)$ & $(0)$ \\
\hline $\mathbf{1 3}$ & $>128(0)$ & $>128(1)$ & $32(19)$ & $(0)$ \\
\hline $\mathbf{1 4}$ & $>128(0)$ & $>128(3)$ & $16(22)$ & $(0)$ \\
\hline $\mathbf{1 5}$ & $>128(0)$ & $>128(3)$ & $16(19)$ & $(0)$ \\
\hline $\mathbf{0 - V a n i l l i n}$ & $>128(0)$ & $16(8)$ & $16(20)$ & $(7)$ \\
\hline Ampicillin & $2(35)$ & $>8(10)$ & - & $(18)$ \\
\hline Fluconazole & - & - & $8(25)$ & - \\
\hline
\end{tabular}

${ }^{a}$ The results obtained from the agar well diffusion method are given in parentheses. 
logarithm of predicted blood/brain barrier partition coefficient, $\log$ B/B (-3.0 to 1.o); apparent Madin-Darby canine kidney cell permeability (PDMCK) that mimic the blood-brain barrier for non-active transport in $\mathrm{nm} \mathrm{s}^{-1}$, PMDCK $(<25$ poor to > 500 great); skin permeability, $\log K_{\mathrm{p}}$ (-8.0 to -1.0$)$; logarithm of binding constant to human serum albumin, $\log K_{\text {HAS }}(-1.5$ to 1.2$)$; qualitative human oral absorption, HOA (1: low, 2: medium, 3: high); percent of $\mathrm{HOA}$ (>80\%: high, $<25 \%$ : poor) (Table 2).

Table 2: Calculated drug-likeness, molecular properties and ADME predictions for Compounds 1-15 using QikProp

\begin{tabular}{|c|c|c|c|c|c|c|c|c|c|c|c|}
\hline 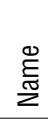 & 㥕 & $\sum_{0}^{\infty}$ & $\begin{array}{l}\text { zo } \\
\text { 잉 } \\
\text { 은 }\end{array}$ & $\begin{array}{l}\text { 吉 } \\
\text { 志 } \\
\text { 으 }\end{array}$ & 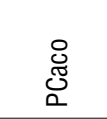 & $\begin{array}{l}\text { ஜ } \\
\text { 으 }\end{array}$ & $\begin{array}{l}\text { 듬 } \\
\sum_{\Delta}\end{array}$ & 음 & $\begin{array}{l}\text { 怘 } \\
\text { 章 } \\
\text { 으 }\end{array}$ & $\begin{array}{l}\text { 또 } \\
\text { 오 }\end{array}$ & $\begin{array}{l}\text { 옹 } \\
\text { 옹 }\end{array}$ \\
\hline 1 & 0 & 0 & 3.25 & -5.62 & 2723.09 & -0.42 & 1460.83 & -0.86 & 0.02 & 3.00 & 100.00 \\
\hline 2 & 0 & 1 & 1.60 & -5.35 & 841.86 & 0.08 & 454.39 & -3.35 & -0.45 & 3.00 & 88.66 \\
\hline 3 & 0 & 1 & 2.30 & -5.49 & 771.51 & 0.04 & 413.49 & -3.43 & -0.15 & 3.00 & 92.10 \\
\hline 4 & 0 & 1 & 2.56 & -5.70 & 863.10 & 0.07 & 466.79 & -3.33 & -0.01 & 3.00 & 94.48 \\
\hline 5 & 1 & 0 & 4.19 & -5.46 & 3553.49 & -0.18 & 3632.38 & -0.66 & 0.32 & 3.00 & 100.00 \\
\hline 6 & 0 & 1 & 4.67 & -5.54 & 4079.69 & 0.05 & 10000.00 & -0.68 & 0.45 & 3.00 & 100.00 \\
\hline 7 & 0 & 0 & 4.30 & -5.60 & 3423.27 & -0.15 & 4640.13 & -0.76 & 0.35 & 3.00 & 100.00 \\
\hline 8 & 1 & 0 & 4.23 & -5.53 & 3421.94 & -0.24 & 2720.15 & -0.67 & 0.26 & 3.00 & 100.00 \\
\hline 9 & 0 & 0 & 4.26 & -5.55 & 3438.21 & -0.20 & 3397.39 & -0.72 & 0.27 & 3.00 & 100.00 \\
\hline 10 & 0 & 1 & 1.74 & -5.21 & 753.41 & 0.05 & 403.01 & -3.45 & -0.35 & 3.00 & 88.62 \\
\hline 11 & 0 & 1 & 2.74 & -5.42 & 845.05 & -0.04 & 456.25 & -3.16 & -0.17 & 3.00 & 95.37 \\
\hline 12 & 0 & 1 & 2.61 & -4.14 & 3405.88 & 0.02 & 4692.47 & -1.47 & -0.19 & 3.00 & 100.00 \\
\hline 13 & 0 & 1 & 0.98 & -6.33 & 110.12 & 0.18 & 55.78 & -5.96 & -0.33 & 2.00 & 69.22 \\
\hline 14 & 0 & -1 & 2.38 & -5.13 & 1254.71 & -0.73 & 632.21 & -1.74 & -0.24 & 3.00 & 96.34 \\
\hline 15 & 0 & 0 & 4.02 & -5.72 & 3376.93 & -0.33 & 1843.40 & -0.61 & 0.25 & 3.00 & 100.00 \\
\hline
\end{tabular}

${ }^{a}$ The assessment uses a knowledge-based set of rules, including checking for suitable values of percent human oral absorption, number of metabolites, number of rotatable bonds, $\log$, solubility and cell permeability.

\section{RESULTS AND DISCUSSION}

Using the conventional method, the proposed Schiff bases of $o$-vanillin were successfully synthesized giving yields between 70-90\%. In the IR spectra, all the compounds had a strong $\mathrm{C}=\mathrm{N}$ stretching band at $1627-1651 \mathrm{~cm}^{-1}$. The ${ }^{1} \mathrm{H}-\mathrm{NMR}$ spectra of the compounds showed that the protons belonging to the compounds in the $\mathrm{HC}=\mathrm{N}$ group were exhibited at $\delta 8.10-8.25 \mathrm{ppm}$ as a singlet, in which both the IR ${ }^{1} \mathrm{H}-\mathrm{NMR}$ data were accepted as evidence for the formation of an imine 
bond. All the other protons were observed according to the expected chemical shift and integral values. The molecular ion peaks $\left(\mathrm{M}^{+}\right)$of the compounds were examined under electron ionization and they confirmed the molecular weights of the compounds. MIC and inhibition zones of Compounds 1-15 were evaluated for their antibacterial and antifungal activities using $O$-vanillin, ampicillin and fluconazole as a standard for the microorganisms (S. aureus, E. coli, C. albicans and $P$. aeruginosa). Throughout the experiments, the parent compound $o$-vanillin was observed to be active for $E$. coli and $C$. albicans but there was minimum or no activity for $S$. Aureus and P. aeruginosa. The best activity of $o$-vanillin was seen against $C$. albicans with the MIC value of $16 \mu \mathrm{g} / \mathrm{mL}$. Since none of the synthesized compounds was found to be active against $P$. aeruginosa during the measurement of the diameter for inhibition zones, further analysis on the compounds was not performed for this microorganism and broth microdilution experiments were not undertaken to determine MIC. According to the results on the inhibition zones of $S$. aureus, Compounds 3, 4, 9, 10, 11 were more active compared to the parent compound $o$-vanillin; however, they were not as active as ampicillin. Similarly, for E. coli, Compound 9 showed similar activity to $o$-vanillin but performed worse than ampicillin. All the compounds had moderate to strong activity against $C$. albicans resulting in similar inhibition zones. In broth dilution experiments, Compounds 1, 2, 3, 8, 10, 12, 14, 15 were found to have an MIC of $16 \mu \mathrm{g} / \mathrm{mL}$, which was similar to that of $o$-vanillin. Another significant result was that Compound 9 had an MIC value of $8 \mu \mathrm{g} / \mathrm{mL}$ presenting the same anti-fungal activity as fluconazole.

In this study, the drug-likeness, molecular and ADME properties of all compounds were promising presenting a drug-like/lead-like profile according to their \#stars rankings. The combinations of HOA values being mostly around 3 (except Compound 13), \%HOA values ranging from 88.62 to $100 \%$, all PCaco values being high and $\log \mathrm{Kp}$ values varying between -0.61 and -5.96 indicate that these compounds can be effectively used in both oral and topical preparations. It is important to note that the binding of drugs to plasma proteins (such as human serum albumin, lipoprotein, glycoprotein, $\alpha, \beta$ and $\gamma$ globulins) greatly reduces the quantity of the drug in general blood circulation. In other words, the less bound a drug is, the more efficiently it can traverse cell membranes or diffuse. Furthermore, the logarithm of the predicted binding constant to human serum albumin, $\log K_{\text {HSA, }}$ values of the synthesized Schiff bases varied between -0.45 to +0.45 , which showed that these derivatives might circulate freely and easily traverse cell membranes. The HERG $\mathrm{K}^{+}$channel, best known for its impact on the electrical activity of the heart by synchronizing beating, appears to be the molecular target responsible for the cardiac toxicity of a wide range of therapeu- 
tic drugs ${ }^{55}$. HERG has also been found associated with modulating the functions of certain cells of the nervous system and with establishing and maintaining cancer-like features in leukemic cells ${ }^{56}$. Thus, HERG $\mathrm{K}^{+}$channel blockers are potentially toxic and the predicted $\mathrm{IC}_{50}$ values often provide reasonable predictions for the cardiac toxicity of drugs in the early stages of drug discovery ${ }^{57}$. The logHERG values of the compounds predicted with the in silico method were between -4.14 and -6.33 with only Compound 13 exhibiting a tendency for the blockage of this channel. As a basic rule, polar drugs cannot easily penetrate blood-brain barrier (BBB). The blood/brain partition coefficient (log BB), PMDCK and $\log P o / w$ values are useful to determine the penetration capacity of a compound from BBB. The values predicted for these parameters of the synthesized compounds were within the ranges defined for 95\% of drugs. Moreover, the predicted CNS value of the compounds was between -1 and +1 indicating mild to medium activity.

Although the prediction results must be checked with actual experiments, the activity studies showed that the Schiff bases derived from $o$-vanillin derivatives could be considered as potential antifungal agents. Furthermore, investigations showed that the Schiff bases synthesized from the starting point of $o$-vanillin can act as a monovalent bidentate ligand, which develop antibacterial properties by combining the azomethine nitrogen and phenolic oxygen atom ${ }^{58}$. Based on this information, we plan to undertake further research to evaluate the metal complexes of these ligands and their antibacterial/antifungal properties.

\section{REFERENCES}

1. Shi, L.; Ge, H. M.; Tan, S. H., et al., Synthesis and Antibacterial Activities of Schiff Bases Derived from 5-Chloro-Salicylaldehyde. Eur. J. Med. Chem. 2007, 42 (4), 558-564.

2. Moss, G. P.; Smith, P. A. S.; Tavernier, D., Glossary of Class Names of Organic Compounds and Reactivity Intermediates Based on Structure (Iupac Recommendations 1995). Pure App. Chem. 1995; Vol. 67, p 1307.

3. Kajal, A.; Bala, S.; Kamboj, S., et al., Schiff Bases: A Versatile Pharmacophore. J. Catal. 2013 , 2013,14 .

4. Eliot, A. C.; Kirsch, J. F., Pyridoxal Phosphate Enzymes: Mechanistic, Structural, and Evolutionary Considerations. Ann. Rev. Biochem. 2004, 73, 383-415.

5. Hernández-Molina, R.; Mederos, A., 1.19 - Acyclic and Macrocyclic Schiff Base Ligands A2 Mccleverty, Jon A. In Comprehensive Coordination Chemistry Ii, Meyer, T. J., Ed. Pergamon: Oxford, 2003; pp 411-446.

6. Nozaki, H.; Takaya, H.; Moriuti, S.; Noyori, R., Homogeneous Catalysis in the Decomposition of Diazo Compounds by Copper Chelates. Tetrahedron. 1968, 24 (9), 3655-3669.

7. Hindson, J. C.; Ulgut, B.; Friend, R. H., et al., All-Aromatic Liquid Crystal TriphenylamineBased Poly(Azomethine)S as Hole Transport Materials for Opto-Electronic Applications. J. Mater. Chem. 2010, 20 (5), 937-944. 
8. Petrus, M. L.; Bein, T.; Dingemans, T. J.; Docampo, P., A Low Cost Azomethine-Based Hole Transporting Material for Perovskite Photovoltaics. J. Mater. Chem. 2015, 3 (23), 12159-12162.

9. Işık, D.; Santato, C.; Barik, S.; Skene, W. G., Charge-Carrier Transport in Thin Films of П-Conjugated Thiopheno-Azomethines. Org. Electron. 2012, 13 (12), 3022-3031.

10. Sicard, L.; Navarathne, D.; Skalski, T.; Skene, W. G., On-Substrate Preparation of an Electroactive Conjugated Polyazomethine from Solution-Processable Monomers and Its Application in Electrochromic Devices. Adv. Func. Mat. 2013, 23 (28), 3549-3559.

11. Uribe-Romo, F. J.; Hunt, J. R.; Furukawa, H., et al., A Crystalline Imine-Linked 3-D Porous Covalent Organic Framework. J. Am. Chem. Soc. 2009, 131 (13), 4570-4571.

12. Sridhar, S. K.; Saravanan, M.; Ramesh, A., Synthesis and Antibacterial Screening of Hydrazones, Schiff and Mannich Bases of Isatin Derivatives. Eur. J. Med. Chem. 2oo1, 36 (7-8), 615-625.

13. Mladenova, R.; Ignatova, M.; Manolova, N., et al., Preparation, Characterization and Biological Activity of Schiff Base Compounds Derived from 8-Hydroxyquinoline-2-Carboxaldehyde and Jeffamines Ed®. Eur. Polym. J. 2002, 38 (5), 989-999.

14. Panneerselvam, P.; Nair, R. R.; Vijayalakshmi, G., et al., Synthesis of Schiff Bases of 4-(4-Aminophenyl)-Morpholine as Potential Antibacterial Agents. Eur. J. Med. Chem. 2oo5, 40 (2), 225-229.

15. Walsh, O. M.; Meegan, M. J.; Prendergast, R. M.; Al Nakib, T., Synthesis of 3-Acetoxyazetidin-2-Ones and 3-Hydroxyazetidin-2-Ones with Antifungal and Antibacterial Activity. Eur. $J$. Med. Chem. 1996, 31 (12), 989-1000.

16. Pandeya, S. N.; Sriram, D.; Nath, G.; DeClercq, E., Synthesis, Antibacterial, Antifungal and Anti-Hiv Activities of Schiff and Mannich Bases Derived from Isatin Derivatives and N-[4-(4'Chlorophenyl)Thiazol-2-Yl] Thiosemicarbazide. Eur. J. Pharm. Sci. 1999, 9 (1), 25-31.

17. Pahontu, E.; Ilies, D. C.; Shova, S., et al., Synthesis, Characterization, Crystal Structure and Antibacterial Activity of Copper(Ii) Complexes with the Schiff Base Derived from 2-Hydroxy4-Methoxybenzaldehyde. Molecules. 2015, 20 (4), 5771-5792.

18. Liu, M. C.; Lin, T. S.; Sartorelli, A. C., Synthesis and Antitumor Activity of Amino Derivatives of Pyridine-2-Carboxaldehyde Thiosemicarbazone. J. Med. Chem. 1992, 35 (20), 3672-3677.

19. Hodnett, E. M.; Mooney, P. D., Antitumor Activities of Some Schiff Bases. J. Med. Chem. 1970, $13(4), 786-786$.

20. Alt, G. H. C. C., MO) Plant Growth Regul. 1980.

21. Hamada, Y.; Takeuchi, I.; Ito, Y., et al., On the Antibacterial Activity and Syntheses of Salicylanilide Derivatives. Yakugaku Zasshi. 1981, 101 (7), 633-641.

22. Ismail, M. M., New Derivatives of 3,5-Dichlorosalicylaldehyde as Antimycotic Agents. Indian J. Pharm. Sci. 1986, 48 (5), 121-124.

23. Abbaspour, A.; Esmaeilbeig, A. R.; Jarrahpour, A. A., et al., Aluminium(Iii)-Selective Electrode Based on a Newly Synthesized Tetradentate Schiff Base. Talanta. 2oo2, 58 (2), 397-403.

24. Mahajan, R. K.; Kaur, I.; Kumar, M., Silver Ion-Selective Electrodes Employing Schiff Base P-Tert-Butyl Calix[4]Arene Derivatives as Neutral Carriers. Sens. Actuators B. Chem. 2oo3, 91 (1-3), 26-31.

25. Ganjali, M. R.; Golmohammadi, M.; Yousefi, M., et al., Novel Pvc-Based Copper(Ii) 
Membrane Sensor Based on 2-(1'-(4'-(1'-Hydroxy-2"-Naphthyl)Methyleneamino)Butyl Iminomethyl)-1-Naphthol. Anal. Sci. 2003, 19 (2), 223-7.

26. Jain, A. K.; Gupta, V. K.; Ganeshpure, P. A.; Raisoni, J. R., Ni(Ii)-Selective Ion Sensors of Salen Type Schiff Base Chelates. Anal. Chim. Acta 2005, 553 (1-2), 177-184.

27. Jeong, T.; Lee, H. K.; Jeong, D.-C.; Jeon, S., A Lead(Ii)-Selective Pvc Membrane Based on a Schiff Base Complex of N,N'-Bis(Salicylidene)-2,6-Pyridinediamine. Talanta. 2005, 65 (2), 543-548.

28. Gupta, V. K.; Singh, A. K.; Mehtab, S.; Gupta, B., A Cobalt(Ii)-Selective Pvc Membrane Based on a Schiff Base Complex of N,N'-Bis(Salicylidene)-3,4-Diaminotoluene. Anal. Chim. Acta 2006, 566 (1), 5-10.

29. Pfeiffer, P.; Breith, E.; Lubbe, E.; Tsumaki, T., Tricyclic Ortho-Condensed Partial Valence Rings. Ann. 1933, 503, 84-130.

30. Hunter, L.; Marriott, J. A., CoöRdinated Copper and Nickel Compounds of Salicylidene Derivatives. J. Chem. Soc. 1937, 2000-2003.

31. Sacconi, L.; Ciampolini, M.; Maggio, F.; Cavasino, F. P., Coordination Chemistry. Ix. Stereochemistry of Some Complex Compounds of Cobalt(Ii) with N-Substituted Salicyl-Aldimines. $J$. Am. Chem. Soc. 1962, 84, 3246-3248.

32. Holm, R. H.; Swaminathan, K., Nickel(Ii) Complexes. Iii. Bis-(N-Arylsalicylaldimine) Complexes. Inorg. Chem. 1962, 1, 599-607.

33. Percy, G. C.; Thornton, D. A., N-Aryl Salicylaldimine Complexes. Infrared and Pmr Spectra of the Ligands and Vibrational Frequencies of Their Metal(Ii) Chelates. J. Inorg. Nucl. Chem. 1972, 34 (11), 3357-3367.

34. Cozzi, P. G., Metal-Salen Schiff Base Complexes in Catalysis: Practical Aspects. Chem. Soc. Rev. 2004, 33 (7), 410-421.

35. Chandra, S.; Sangeetika, Epr and Electronic Spectral Studies on Copper(Ii) Complexes of Some N-O Donor Ligands. J. Indian Chem. Soc. 2004, 81 (3), 203-206.

36. Burt, S., Essential Oils: Their Antibacterial Properties and Potential Applications in Foods a Review. Int. J. Food Microbiol. 2004, 94 (3), 223-253.

37. Davidson, P. M.; Naidu, A. S. In Phyto-Phenols, CRC Press LLC, Boca Raton, 2ooo, pp 265-294.

38. Holley, R. A.; Patel, D., Improvement in Shelf-Life and Safety of Perishable Foods by Plant Essential Oils and Smoke Antibacterials. Food Microbiol. 2005, 22 (4), 273-292.

39. Shazia, M.; Saba, M.; Jasmine, F., et al., Anti-Bacterial, Anti-Oxidant and Cytotoxic Potential of Various Extracts of Myristica Fragrans. Int. J. Res. Ayurveda Pharm. 2015, 6 (5), 643-648.

40. Fitzgerald, D. J.; Stratford, M.; Gasson, M. J., et al., Mode of Antibacterial Action of Vanillin against Escherichia Coli, Lactobacillus Plantarum and Listeria Innocua. J. Appl. Microbiol. 2004, 97 (1), 104-113.

41. Leifertova, I.; Hejtmankova, N.; Hlava, H., et al., Antifungal and Antibacterial Effects of Phenolic Substances. A Study of the Relation between The Biological Activity and the Constitution of the Investigated Compounds. Acta Univ. Palacki. Olomuc., Fac. Med. 1975, 74, 83-101.

42. Ohmagari, H.; Fukahori, A.; Nakaya, M., et al., Crystal Structures and Magnetic Properties of Manganese(Iii) Complexes with Tridentate Schiff Base Ligands. J. Inc. Phenom. Macrocycl. 
Chem. 2015, 82 (1), 213-218.

43. Petek, H.; Albayrak, C.; Iskeleli, N. O., et al., Crystallographic and Conformational Analyses of Zwitterionic Form of (E)-2-Methoxy-6-[(2-Morpholinoethylimino)Methyl]Phenolate. $J$. Chem. Crystallogr. 2007, 37 (4), 285-290.

44. Wang, Y.-X.; Zhao, W.-L.; Bi, C.-W., et al., Synthesis and Structure-Activity Relationship of N-(2-Arylethyl) Isoquinoline Derivatives as Anti-Cancer Agents. Yao Xue Xue Bao. 2012, 47 (2), 200-205

45. Sasmal, S.; Mohanta, S., M-Phenoxo-M-Pseudohalide and M-Pseudohalide Dinuclear, Tetranuclear and One-Dimensional Complexes: Magneto-Structural Correlation and Interesting Type of Solid State Isomerism. J. Chem. Sci. (Bangalore, India) 2012, 124 (6), 1353-1364.

46. Mistri, S.; Puschmann, H.; Manna, S. C., DNA/Protein Binding, Cytotoxicity and Catecholase Activity Studies of a Piperazinyl Moiety Ligand Based Nickel(Ii) Complex. Polyhedron. 2016, 115, 155-163.

47. Wang, Y.-X.; Li, Y.-H.; Li, Y.-H., et al., Synthesis, Structure-Activity Relationship and in Vitro Biological Evaluation of N-Arylethylisoquinoline Derivatives as Coxsackievirus B3 Inhibitors. Bioorg. Med. Chem. Lett. 2011, 21 (19), 5787-5790.

48. Das, K.; Datta, A.; Liu, P.-H., et al., Structural Characterization of Cobalt(Ii) Complexes of an N,O Donor Schiff Base and Their Activity on Carcinoma Cells. Polyhedron. 2014, 71, 85-90.

49. Shit, S.; Sankolli, R.; Row, T. N. G., A Dinuclear Cadmium(Ii) Schiff Base Thiocyanato Complex: Crystal Structure and Fluorescence. Acta Chim. Slov. 2014, 61 (1), 59-66.

50. Perez, C.; Pauli, M.; Bazerque, P., An Antibiotic Assay by Agar-Well Diffusion Method. Acta Biol. Med. Exp. 1990, 15, 113-115.

51. Ahmad, I.; Mehmood, Z.; Mohammad, F., Screening of Some Indian Medicinal Plants for Their Antibacterial Properties. J. Ethnopharmacol. 1998, 62 (2), 183-93.

52. Balouiri, M.; Sadiki, M.; Ibnsouda, S. K., Methods for in Vitro Evaluating Antibacterial Activity: A Review. J. Pharm. Anal. 2016, 6 (2), 71-79.

53. (CLSI), C. L. S. I., Performance Standards for Antibacterial Susceptibility Testing TwentyThird Informational Supplement. Clsi Document. M10o-S20. 2013.

54. (CLSI), C. L. S. I., Methods for Dilution Antibacterial Susceptibility Tests for Bacteria That Grow Aerobically; Approved Standard-Ninth Edition, Clsi Document: Mo7-A9. 2012.

55. Vandenberg, J. I.; Walker, B. D.; Campbell, T. J., Herg K+ Channels: Friend and Foe. Trends Pharmacol. Sci. 2001, 22 (5), 240-246.

56. Chiesa, N.; Rosati, B.; Arcangeli, A., et al., A Novel Role for Herg K+ Channels: Spike-Frequency Adaptation. J. Physiol. (Cambridge, U. K.) 1997, 501 (2), 313-318.

57. Aronov, A. M., Predictive in Silico Modeling for Herg Channel Blockers. Drug Discov. Today. 2005, 10 (2), 149-155.

58. Anupama, B.; Sunita, M.; Shiva Leela, D., et al., Synthesis, Spectral Characterization, DNA Binding Studies and Antibacterial Activity of Co(Ii), Ni(Ii), Zn(Ii), Fe(Iii) and Vo(Iv) Complexes with 4-Aminoantipyrine Schiff Base of Ortho-Vanillin. J. Fluoresc. 2014, 24 (4), 1067-1076.

(Received 14 September 2016; accepted o6 December 2016) 